\title{
CIRUGÍA ROBÓTICA: UNA MIRADA HACIA EL FUTURO.
}

\author{
Juan I. Martínez-Salamanca' y Carlos Hernández².
}

'Robotic Prostatectomy Program \& Urology Oncology Outcomes. Weill Medical College at Cornell University. New York Presbyterian Hospital. Brady Urological Institute. New York, USA

2Servicio de Urología. Hospital General Universitario "Gregorio Marañón". Madrid. (España).

Resumen.- La cirugía robótica se encuentra en plena expansión. El objetivo de toda tecnología sanitaria nueva es mejorar los resultados en el tratamiento de las diferentes patologías. Aventuramos nuestra perspectiva de futuro tanto del desarrollo de la actual generación de robots quirúrgicos como de las nuevas mejoras que se le podrían incorporar a corto y medio plazo.

Palabras clave: Cirugía robótica. Futuro. Nuevas tecnologías.

\begin{abstract}
Summary.- The surgery robotics is in full expansion. The objective of all new sanitary new technology is to improve the results in the treatment of the different pathologies. We believe that our of future perspective both in the development of the current generation of surgical robots and in the improvements that could be incorporated to short and half term.
\end{abstract}

Keywords: Robotic surgery. Future. Perspective. New technologies.

\section{INTRODUCCIÓN}

"La mejor forma de predecir el futuro, es invertarlo"

Alan Kay

Estamos viviendo una verdadera convulsión en nuestra especialidad con la expansión de la cirugía robótica. Según datos recientes, el número de unidades da Vinci® en funcionamiento supera las 550. Casi 100 de ellas en Europa, pero la pregunta es, ¿̇cuál será el futuro a medio y largo plazo de esta tecnología? La respuesta sin duda es desconocida, pero intentaremos vislumbrar algunas posibles opciones.

Debemos considerar que este futuro engloba dos parcelas. La primera de ellas se refiere a qué va a pasar con la cirugía robótica tal y como hoy la entendemos. Es decir, los sistemas maestro-esclavo y más concretamente la actual generación da Vin$\mathrm{ci} \otimes$ en sus versiones de tres, cuatro brazos o "S". 
En segundo lugar, qué modificaciones o mejoras se pueden añadir en el futuro al sistema actual para mejorarlo o incluso la aparición de otros sistemas.

\section{Futuro del actual sistema da Vinci®}

La ventajas que ha demostrado claramente la cirugía robótica sobre la cirugía abierta provienen de dos fuentes; las heredadas de la cirugía laparoscópica convencional (menor pérdida sanguínea, incisiones mas pequeñas y recuperación postoperatoria más rápida) y las propias (visión tridimensional, magnificación, sencillez y precisión en los movimientos).

En nuestra especialidad tan sólo disponemos de datos y no del todo concluyentes a largo plazo, sobre la prostatectomía radical. Son muchas las técnicas que se están realizando mediante este abordaje, pero aún los resultados no están maduros. Por tanto, nos encontramos en el camino y nuestra meta será demostrar o negar las ventajas, en cuanto a mejores resultados se refiere, sobre las técnicas anteriores. De esto dependerá principalmente su definitiva instauración y que se llegue a convertir en el Standard de tratamiento de muchas patologías. Precisamente en este sentido, existe una creciente preocupación en la comunidad científica internacional. Es importante disponer de adecuados instrumentos de medida para objetivar los resultados. Debemos intentar que no suceda, como con otras tecnologías anteriores, que la presión comercial o el entusiasmo inicial, no permita realizar buenos estudios prospectivos, aleatorizados e independientes para poder validar verazmente las hipótesis. La ética y compromiso en el tratamiento de nuestros enfermos esta en juego. Esto es tarea de todos.

Por otro lado, si se confirmará su superioridad sobre las técnicas vigentes en determinadas patologías, la técnica debería implantarse en la mayoría de centros o al menos en centros de referencia que atesoraren la mayor experiencia. Para ello, hoy en día un problema importante, radica en el elevado coste del aparato así como su mantenimiento y fungibles.

Lleva casi una década funcionando, y la siempre esperada reducción de costes no se ha producido, si cabe se han incrementado. Quizás en un momento determinado se deberá ejercer presión, desde las instituciones, para que esto cambie. La aparición de competencia al actual monopolio, probablemente haría bajar los precios y aumentar la calidad.

Concretamente en nuestro Sistema Público de Salud la introducción de esta tecnología se esta realizando de una manera lenta debido a su elevado precio. Muchas son las consideraciones a favor y en contra, veremos lo que nos deparará el futuro (1).

\section{Mejoras y conceptos técnicos futuros}

En el año 2004 el grupo de Instituto Montsouris (2) señalaba cuales deberían ser las mejoras del sistema da Vinci® original. Eran las siguientes:

- Instrumentos de $5 \mathrm{~mm}$ en vez de los originales de 8 $\mathrm{mm}$, hecho este que ya se ha hecho realidad y están disponibles en el mercado. El sistema de movimiento permite su introducción a través de una endoscopio flexible (gastroscopio o cistoscopio) lo que dará lugar muy pronto a la "cirugía endocavitaria compleja" a través de orificios naturales.

- La incorporación de un cuarto brazo controlado por el cirujano, hecho este que también ya es realidad. Puede ahorrar un ayudante y en algunos procedimientos podría permitir la cirugía con un "único cirujano". Situación esta con una parte negativa desde el punto de vista del entrenamiento y la formación.

- Disminución de tamaño del equipo tanto de la consola como del carro quirúrgico. Actualmente es tremendamente pesado y difícil de transportar. Probablemente si esto se lleva a cabo, se podrían instalar los brazos en el techo del quirófano, como un elemento más del mismo (3). El acople al paciente sería mucho más sencillo y natural y en caso de una emergencia acortaría el tiempo para desacoplar el sistema y reconvertir la cirugía.

- La cámara con dos ópticas que permite generar una imagen tridimensional podría ser mejorada si incorporase una tercera óptica (canal) a modo de "ojo de pez". Esto permitiría una visión estereoscópica tridimensional y panorámica.

Además de estas consideraciones presentes y futuras, la tecnología robótica es el escenario perfecto para el desarrollo de la simulación y navegación quirúrgica mediante la integración de los modernos sistemas de imagen radiológicos (TC y RMN multicorte) que permiten generar reconstrucciones tridimensionales de gran calidad y precisión (4). Podría tener varias aplicaciones:

- Entrenamiento mediante simulación preoperatoria de cada paciente sobre sus imágenes reales. Es decir, el cirujano podría realizar la cirugía en un simulador antes de realizársela al paciente con las referencias que se encontrará cuando lo haga. Esto puede aportar unas posibilidades inmensas, tanto para optimizar y asegurar los resultados como para fines de entrenamiento.

- Posibilidad de integración de las imágenes a tiempo real en la consola quirúrgica y que el sistema guíe al 
cirujano hacia sus objetivos anatómicos concretos de una manera precisa.

- La adición al sistema de dispositivos que permitan el reconocimiento de tejidos a tiempo real (microscopio multifotónico (5-7), tomografía de coherencia óptica (8-10). Esto permitiría al cirujano reconocer fácilmente las estructuras a preservar o cuáles por el contrario debe extirpar.

Una de las mayores limitaciones que presenta esta generación de sistemas robóticos es la ausencia de sensación táctil (háptica) (3). Es decir el cirujano no recibe ninguna información a través de sus manos que su cerebro pueda integrar sobre la fuerza o la consistencia del tejido que esta manejando. En ocasiones la posibilidad de palpar un tejido o un órgano es útil, así como por ejemplo saber cuanta fuerza ejerces sobre un hilo de sutura (11).

Estudios recientes han revelado que la falta de sensación táctil durante la cirugía asistida por robot puede llevar a un aumento en la agresión tisular y a un daño no intencionado del mismo, y los cirujanos a los que se les ha provisto de sensación de la fuerza ejercida, han mejorado considerablemente sus resultados $(12,13)$. Hay varios centros trabajando en investigación y desarrollo sobre este tema (Ver Capítulo 4, Equipamiento y Tecnología en Cirugía Robótica).

Existen simuladores robóticos que proveen la sensación de fuerza y tensión de las estructuras manejadas. Se conoce como sistema THUMP (14).

Recientemente una compañía llamada Mako Surgical Corp (Ft. Lauderdale, FL) a lanzado al mercado su sistema Haptic Guidance System (HGS®) para realizar artroplastias de rodilla mediante un brazo robótico manejado directamente por el cirujano. Este sistema permite saber que dureza tiene el tejido sobre el que se esta operando, es decir, proporciona retroalimentación háptica al cirujano. (www.makosurgical.com ). Probablemente las futuras generaciones de robot quirúrgicos puedan incorporar esta función.

\section{CONCLUSIONES}

Como se puede comprobar estamos en los inicios de un viaje apasionante. Esperemos que la tecnología de la que disponemos y las innovaciones que están por venir, nos ayuden a perfeccionar y optimizar la técnica quirúrgica. Asimismo deseamos que esta mejora en el desarrollo de los procedimientos impacte de manera directa sobre los resultados de nuestros pacientes, haciéndolos cada vez mejores.

\section{BIBLIOGRAFÍA y LECTURAS RECOMENDADAS ( ${ }^{*}$ lectura de interés $y^{* *}$ lectura fundamental)}

*1. MARTINEZ-SALAMANCA, J.; ALLONA, A.: "Razones técnicas y económicas para la implantación de la cirugía robótica en un sistema público de salud (Modelo Español)". Actas Urol. Esp., Monográfico sobre Cáncer de Próstata (En prensa), 2007.

**2. CATHELINEAU, X.; ROZET, F.; VALLANCIEN, G.: "Robotic radical prostatectomy: the European experience”. Urol. Clin. North Am., 31: 693, 2004.

3. KITAGAWA, M. y cols.: "Effect of sensory substitution on suture manipulation forces for surgical teleoperation". Stud. Health Technol. Inform., 98: 157, 2004.

4. COSTE-MANIERE, E. y cols.: "Planning, simulation, and augmented reality for robotic cardiac procedures: The STARS system of the ChIR team". Semin. Thorac. Cardiovasc. Surg., 15: 141, 2003.

5. WEBB, S.E. y cols.: "Multidimensional single-molecule imaging in live cells using total-internal-reflection fluorescence microscopy". Opt. Lett., 31: 2157, 2006.

6. WILLIAMS, R.M.; ZIPFEL, W.R.; WEBB, W.W.: "Multiphoton microscopy in biological research". Curr. Opin. Chem. Biol., 5: 603, 2001.

7. ZIPFEL, W.R. y cols.: "Live tissue intrinsic emission microscopy using multiphoton-excited native fluorescence and second harmonic generation". Proc. Natl. Acad. Sci. USA, 100: 7075, 2003.

8. D'AMICO, A.V. y cols.: "Optical coherence tomography as a method for identifying benign and malignant microscopic structures in the prostate gland". Urology, 55: 783, 2000.

9. FRIED, N.M. y cols.: "Imaging the cavernous nerves in the rat prostate using optical coherence tomography". Lasers Surg. Med., 39: 36, 2007.

10. LI, H. y cols.: "Feasibility of interstitial Doppler optical coherence tomography for in vivo detection of microvascular changes during photodynamic therapy”. Lasers Surg. Med., 38: 754, 2006.

11. BETHEA, B.T. y cols.: "Application of haptic feedback to robotic surgery". J. Laparoendosc. Adv. Surg. Tech. A., 14: 191, 2004.

12. WAGNER, C.; STYLOPOULOS, N.; HOWE, R.: "The role of force feedback in surgery: Analysis of blunt dissection". IEEE 10th Symp. on haptic interfaces for virtual envir\&teleoperator systems, 2002.

13. DEML, B.; ORTMAIER, T.; SEIBOLD, U.: “The touch, and feel in minimally invasive surgery". Workshop on haptic audio visual environments, and their applications, Ottawa, Ontario, 2005.

14. NIEMEYER, G. y cols.: "THUMP: an immersive haptic console for surgical simulation and training". Stud. Health Technol. Inform., 98: 272, 2004. 